\title{
Towards a Knowledge-Intensive Framework for Top-Down Design Context Definition
}

\author{
Nicolas Petrazoller ${ }^{\star}$, Frédéric Demoly, Samuel Deniaud, and Samuel Gomes \\ IRTES-M3M, \\ Université de Technologie de Belfort-Montbéliard (UTBM), \\ 90010 Belfort Cedex, France \\ \{nicolas.petrazoller,frederic.demoly, samuel.deniaud, samuel.gomes\}@utbm.fr
}

\begin{abstract}
This paper presents a skeleton-based modeling approach enabling the definition of a knowledge-intensive design context at the beginning of the embodiment design stage. The research introduces an analogy to the incubator concept by creating a suitable support along the design phase including CAD modeling. The main objective of the proposed approach is to integrate engineering information and knowledge in the early phases of the product development process in a top-down and seamless manner so as to provide a knowledge-based design context for designers. The fact of including a design context in the embodiment design phase will assist designers to make better-informed decisions and therefore linking what (technical entities and engineering data), why (rationale) and how (processes and functions). The concept of design incubator will be defined according to its function, behavior and structure (i.e. skeleton entities, functional surfaces, design spaces, parameters, knowledge and design requirements). The proposed design incubator ensures the knowledge delivery and engineering support at the right time. A case study has been carried out to demonstrate the developed method.
\end{abstract}

Keywords: Assembly modeling, Skeleton-based modeling, Top-down assembly design, Proactive engineering, Design context definition, Knowledge-intensive design.

\section{Introduction}

Nowadays the globalized competitive context requires companies to deliver new products which are more innovative, more efficient with shorter lead time and optimized costs in order to fulfill customer's requirements. Designing such systems requires a phase of architectural design, lead by product architects, which must take into account a growing number of constraints (increased reliability, sustainability, reduced environmental impact, etc.). In addition, since product development includes a large amount of viewpoints [7, product architects have a global view on the system to be developed, especially on functional, structural, behavioral, geometric and physical aspects.

\footnotetext{
^ Corresponding author.
} 
In a context of large scale company with many stakeholders working collaboratively and remotely on the same product, the definition of a design context to assist designers on their collaborative design work is important and critical. Based on previous research works related to assembly design - such as proactive design for assembly and skeleton-based modeling approaches ([8] [9] [10]) -, the main objective of this paper is to propose a proactive top-down modeling approach of design layout elements based on an analogical reasoning approach with incubator in the embodiment design stage. Here, incubator is composed of several layers of engineering information and knowledge (i.e. skeleton entities, functional surfaces, design spaces, parameters, knowledge and requirements to name a few), in order to support designers activity with a well-defined design context. For instance, the flow of engineering information and knowledge, which is required to convert product architect intents into design support elements for designers, can be seen with the analogy of an incubator (i.e. knowledge-intensive and living design context) offering an optimal environment to an embryo (i.e. design concept) until its complete development (i.e. detailed design). This novel approach will support designers by integrating engineering information and knowledge in embodiment design stage, so that designers have all needed inputs and associated procedures to define geometry. Compared to previous research efforts in this field [9], this approach will introduce new technical entities to already defined assembly skeletons, such as interface skeletons and functional surfaces in a top-down and proactive manner.

Built on this, section 2 reviews some previous works in the field of layout modeling, assembly modeling, and geometric skeleton-based modeling. In Section 3 , a terminology of the design incubator concept is proposed and the overall approach is presented. Then section 4 discusses about the deployement of the approach through a mechanical assembly. Finally, conclusions and future work are addressed.

\section{Related Works}

This section states a brief overview of published research works on layout modeling, assembly modeling, and geometric skeleton-based modeling issues, so as to provide the foundation of the proposed approach.

The design phase of a product is composed of several stages [15] from the identification of customer needs to the detailed definition of the product. The approach of this paper will focus on the embodiment design stage of the product, in which layout design has a very important role to play [3]. The using of layout elements composed of geometry and engineering information needed to design permits to support designers and offers the possibility to exchange design data with other teams. From the literature, many attempts have been made to carry out various aspects of the layout design, such as the determination of kinematics constraints between functional components [12], the assessment of design scenarios [1], the deployment of tools based to carry out collaborative design activities among multi-disciplinary teams 2], the capture of all the feasible designs to find an optimal geometry with integration of user-defined constraints 
13, the development of full comprehensive models for spatial constraints and in particular for free-space requirements [18] and even the analysis of interferences between product components 4 .

Literature has provided numerous published research works about Design for assembly (DFA) which seems to be the most investigated component of Design for (DFX). First published contributions introduced heuristic rules and design guidelines as qualitative evaluation (1) 16, 14 [19). More recently, Stone et al. introduced a conceptual DFA method using a functional basis and heuristic rules [17, and a DFA approach has been initiated based on System Modeling Language (SysML) in the PLM context considering an assembly oriented product structure based on preliminary assembly sequence 5. Furthermore, recent research efforts in proactive DFA have proven that the early generation of admissible assembly sequences during conceptual design stages can be created in order to provide an appropriate contextual support for assembly design and modeling phases ( 6 ] [8]), even for the geometric definition in a top-down way 9 .

\section{Proposed Approach}

This section presents the proposed approach which introduces the design incubator concept. Design incubator provides a knowledge-intensive and living support to designers by defining layout product geometry at the beginning of the embodiment design phase. Such analogical reasoning will enable the introduction of a novel paradigm in CAD modeling stage.

\subsection{Terminology}

Based on the SKeLeton geometry-based Assembly Context Definition (SKLACD) approach [9], new geometric elements are introduced and defined so as to clarify the structure of the design incubator (Fig. 10):

- Assembly skeleton entity: This entity is a support for the product modeling phase (line, point, etc.) and can be considered as the first geometric elements to which the designers can allocate and define part volume and geometry.

- Skeleton interface entity: This entity describes some geometric boundaries (circle, square, etc.) which is used to build a functional surface and is supported by an assembly skeleton entity (line, plane, etc.).

- Functional surface entity: Functional surfaces are determined from the kinematic relations and product functionalities. They are limited in space by contours (i.e. skeleton interface entities)

- Design space: Design spaces are used to represent product components in a layout. Their location and orientation in space is represented by a local coordinate frame, to which a simple geometry (cylinder, cube, etc.) is attached [4]

- Design incubator: Design incubator is a set of geometric entities (skeleton assembly entities, skeleton interface entities, design spaces, parameters), knowledge and requirements which are linked formally and semantically.

Fig. 1 presents an UML class diagram of the design incubator concept. 


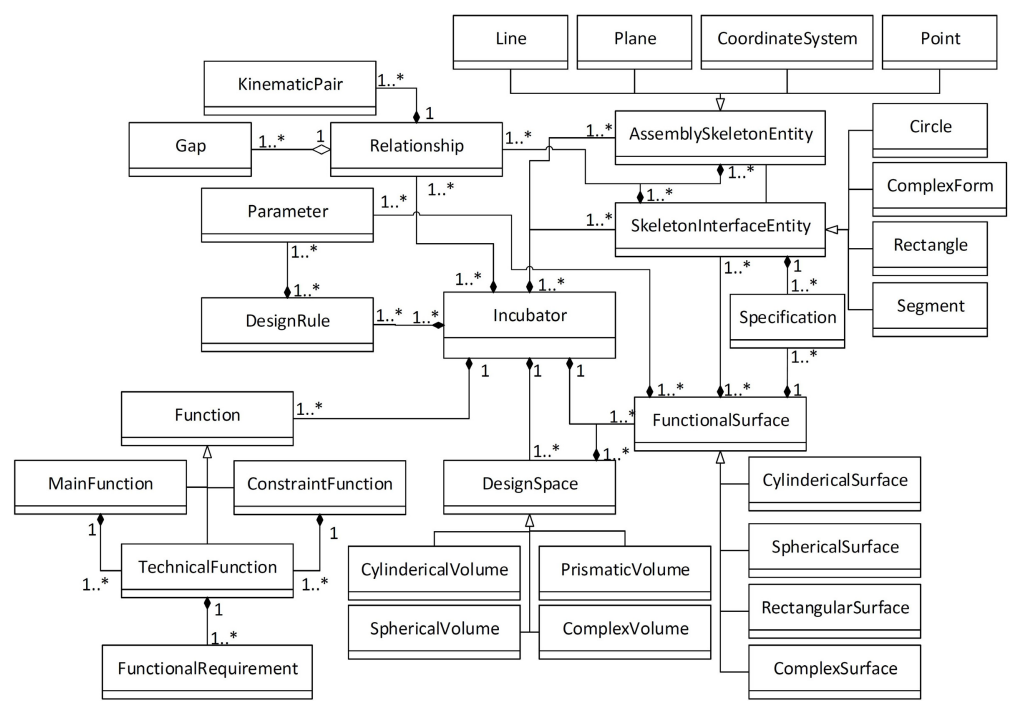

Fig. 1. UML class diagram of the incubator concept

\subsection{Overall Methodology Description}

Based on the SKL-ACD approach [9, an enriched flow chart is introduced to describe the proposed approach in a more detailed view (Fig. 2). An explanation of the different steps of the method is visible below:

- Start: Starting from the early defined assembly sequence generated by the ASDA algorithm and product relational information (contact and precedence information) embedded in graphs and matrices, the product architect defines kinematics/technological pairs in the directed graph;

- Steps 1 and 2. The product structure is automatically generated and assembly skeleton places are assigned inside (Steps 1 and 2 of Fig. 2);

- Steps 3 and 4 . Based on these relationships, assembly constraints are automatically defined and geometric skeleton entities can be generated in order to provide interface control elements for assembly modeling (Steps 3 and 4 of Fig. 21);

- Step 5. The product architect introduces new assembly constraints between the generated geometric skeleton entities, consistent with previously defined kinematic and technological pairs (Step 5 of Fig. 2); A new graph, called skeleton graph, built upon these constraints, is defined by skeleton entities and their related assembly constraints;

- Step 6. This graph is simplified later on by the generalization and the concatenation of skeleton elements into a minimal skeleton graph (Step 6 of Fig. 21);

- Step 7. Based on this minimal skeleton graph and the early-defined assembly sequence, this step allows the structuring and regrouping of skeleton elements in assembly skeletons (Step 7 of Fig. 2); 
- Step 8. An assembly coordinate system is then defined for each assembly layer and associated to each identified base part. Therefore new constraints are introduced to link the defined assembly coordinate systems with the interface control elements from the minimal skeleton graph (Step 8 of Fig. 2);

- Step 2. The resulting assembly skeletons can be allocated to the initial product structure. At this stage, it is possible to assign rights to a skeleton entity which is at the interface of different assembly skeletons (Step 2 of Fig. 2);

- Step 9. Based on the kinematic pairs between components and product architect choice, the skeleton interfaces entities are defined (Step 9 of Fig. 2);

- Step 10. Supported by skeleton interfaces entities, functional surfaces are defined (Step 10 of Fig. 2);

- End: As a result, the assembly skeleton CAD model and functional surfaces are semi-automatically generated.

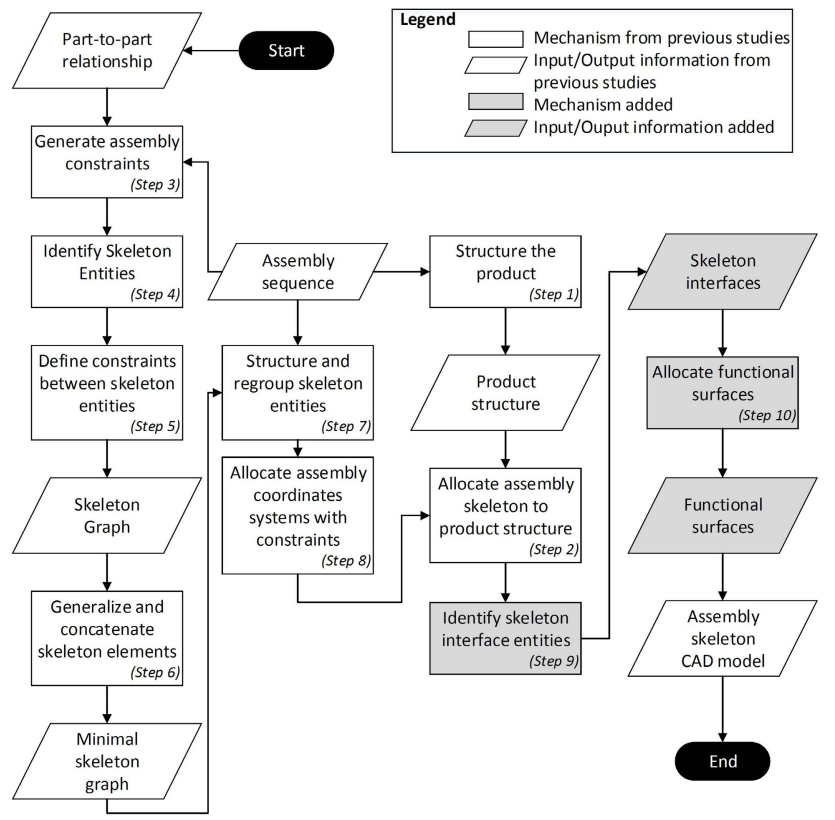

Fig. 2. Enriched flowchart of the proposed approach

\section{Case Study}

In this section, the proposed approach is illustrated with a mechanical system composed of 3 parts. Each step of the method are described for a better understanding (Fig. 33). 


\subsection{Determination of the Skeleton Minimal Graph (Step 3 to 6 of Fig. 2)}

The product architect starts by defining the kinematic pairs between each parts. Based on the kinematic pairs, the skeleton entities and position constraints between each parts are deducted. At this point, to facilitate the management of the skeleton entities, it is possible to simplify the proposed skeleton graph by generalize and concatenate skeleton entities (Fig. 31).

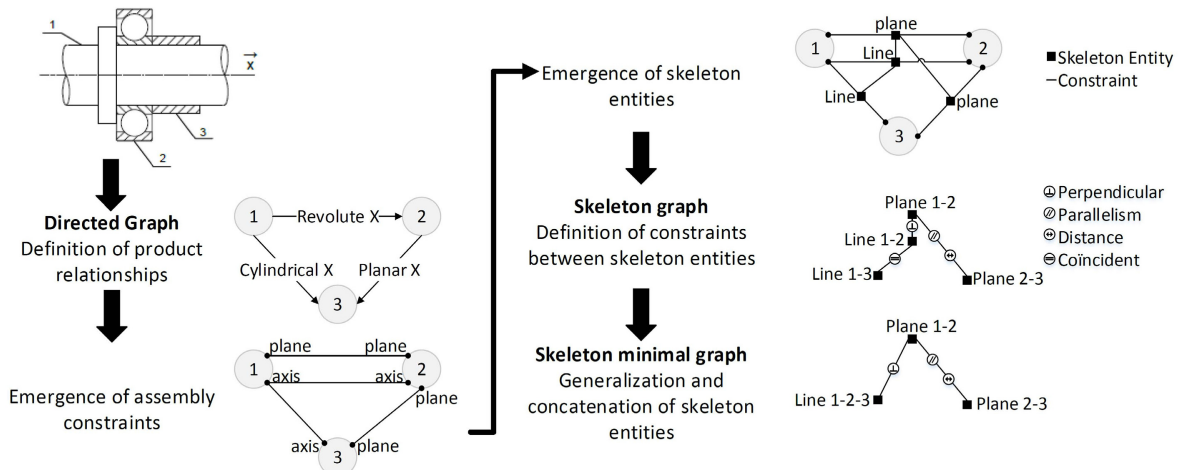

Fig. 3. Definition process of a minimal skeleton model

\subsection{Introduction of an Assembly Coordinate System (Step 7 to 8 of Fig. 2)}

Based on the minimal skeleton graph of the previous step, an assembly coordinate system is introduced. Each skeleton entities are linked to this new assembly coordinate system by the intermediate of geometrical constraints. Then, the geometrical constraints are concatenated (Fig. (4)).

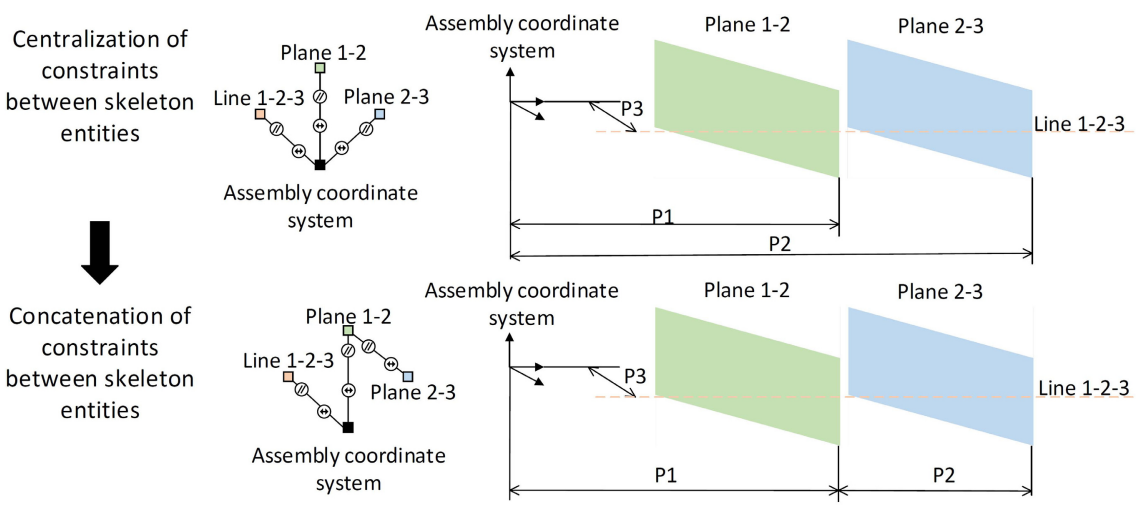

Fig. 4. Impact of the introduction of an assembly coordinate system in the minimal skeleton model 


\subsection{Allocation of Skeleton Interface Entities and Functional Surfaces (Step 9 to 10 of Fig. 2)}

Based on previous steps and Table 1 the skeleton interface entities are defined and associated to a skeleton entity. Then, it is possible to concatenate the skeleton interface entities in order to simplify the design environment. Finally, after this simplification, the functional surface are deduced from the skeleton interface entities (Fig. 5).

Table 1. Definition of the Skeleton interface entities and functional surfaces based on kinematics pairs

\begin{tabular}{|c|c|c|c|c|}
\hline $\begin{array}{l}\text { Kinematic } \\
\text { Pair }\end{array}$ & SKL Entity & SKL Interface Entity & Position & Functional surface \\
\hline Rigid & $\begin{array}{l}\text { Coordinate } \\
\text { system }\end{array}$ & non-determined form & To be defined & To be defined \\
\hline Revolute & Line, Plane & $\begin{array}{l}2 \text { Circles for the plane contact } \\
1 \text { circle for the rotational guidance } \\
1 \text { segment for the rotational guidance non- } \\
\text { determined form }\end{array}$ & $\begin{array}{l}\text { Plan } \\
\text { to be defined } \\
\text { Line }\end{array}$ & $\begin{array}{l}1 \text { disk inside the plane } \\
1 \text { cylindrical surface perpendicular to the plane }\end{array}$ \\
\hline Prismatic & Line, Plane & $\begin{array}{l}\text { non-determined form } \\
1 \text { segment }\end{array}$ & $\begin{array}{l}\text { Plan } \\
\text { Line }\end{array}$ & non-dertermined form perpendicular to the plane \\
\hline Screw & Line, Plane & $\begin{array}{l}1 \text { circle for stoping the thread } \\
1 \text { circle for the rotational guidance } \\
1 \text { segment for the rotational guidance }\end{array}$ & $\begin{array}{l}\text { Plan } \\
\text { to be defined } \\
\text { Line }\end{array}$ & $\begin{array}{l}1 \text { disk inside the plane } \\
1 \text { cylindrical surface perpendicular to the plane }\end{array}$ \\
\hline Cylindrical & Line & $\begin{array}{l}1 \text { circle for the rotational guidance } \\
1 \text { segment for the rotational guidance }\end{array}$ & $\begin{array}{l}\text { To be defined } \\
\text { Line }\end{array}$ & 1 cylindrical surface cocident with the axis \\
\hline Spherical & Point & 1 circle & to be defined & 1 sphere with the center cocident with the point \\
\hline Planar & Plane & non-determined form for the contact & Plan & non-dertermined form inside the plane \\
\hline Point-contact & Point, Plane & non-determined form for the contact & Plan & non-dertermined form inside the plane \\
\hline Line-contact & Line, Plane & non-determined form for the contact & Plan & non-dertermined form inside the plane \\
\hline Curve-contact & Curve & $\begin{array}{l}1 \text { circle } \\
1 \text { segment }\end{array}$ & $\begin{array}{l}\text { To be defined } \\
\text { Line }\end{array}$ & 1 cylindrical surface cocident with the curve \\
\hline
\end{tabular}

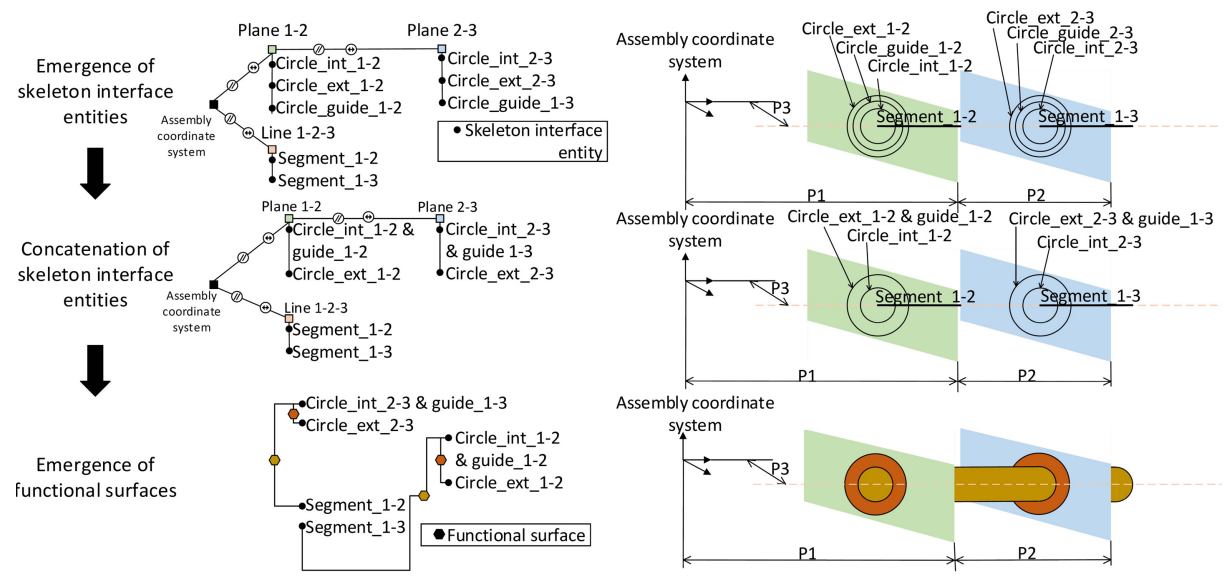

Fig. 5. Generation of the functional surfaces from skeleton interface entities 


\section{Conclusions and Future Work}

In this paper, a method to define a design context in the early phase of the design development process has been presented. The current issue of the paper is to allocate further information to the assembly skeleton model such as skeleton interfaces and functional surfaces. Defining functional surface allocation based on skeleton modeling will permit a better understanding of "what to design" by designers. To illustrate the feasibility and the relevance of the proposed approach, a use case has been carried out. Finally, four main issues demand further research: the allocation of design space, the incorporation of knowledge; adding requirements; and create a link between the method and PLM softwares.

Acknowledgments. The research activity is part of the INGéPROD (Productiveness for Product-Process Engineering in a Design Chain context), which has been funded by French Automotive Cluster Pôle de Compétitivité Véhicule du Futur. The authors would like to thank General Electric for this collaboration and all the financial supports of this research and technology program: DRIRE de Franche-Comté, Communauté dAgglomération du Pays de Montbéliard, Conseil Général du Doubs and Conseil Régional de Franche-Comté.

\section{References}

1. Andreasen, M.M., Kahler, S., Lund, T.: Design for assembly. IFS Publications Ltd., Springer, Verlag, UK (1983)

2. Bai, Y.W., Chen, Z.N., Bin, H.Z., Hu, J.: Collaborative design in product development based on product layout model. Robotics and Computer-Integrated Manufacturing 21, 55-65 (2005)

3. Chen, X., Gao, S., Yang, Y., Zhang, S.: Multi-level assembly model for top-down design of mechanical products. Computer-Aided Design 44, 1033-1048 (2012)

4. Csabai, A., Stroud, I., Xirouchakis, P.C.: Container spaces and functional features for top-down 3D layout design. Computer-Aided Design 34, 1011-1035 (2002)

5. Demoly, F., Gomes, S., Eynard, B., Sagot, J.C.: Towards a design for assembly approach based on SysML paradigm and PLM systems. In: Proc. Second CIRP Int. Conf. Assem. Technol. Syst. (CATS), Toronto, Canada, September 21-23, pp. 100-113 (2008)

6. Demoly, F., Gomes, S., Eynard, B., Rivest, L., Sagot, J.C.: Assembly-oriented product structure based on preliminary assembly process engineering. In: Proc. Int. Conf. Eng. Des., ICED 2009, Stanford, CA, USA, August 24-27 (2009)

7. Demoly, F., Monticolo, D., Eynard, B., Rivest, L., Gomes, S.: Multiple viewpoint modelling framework enabling integrated product process design. International Journal on Interactive Design and Manufacturing 4, 269-280 (2010)

8. Demoly, F., Yan, X.-T., Eynard, B., Rivest, L., Gomes, S.: An Assembly oriented design framework for product structure engineering and assembly sequence planning. Robotics and Computer-Integrated Manufacturing 27, 33-46 (2011)

9. Demoly, F., Toussaint, L., Eynard, B., Kiritsis, D., Gomes, S.: Geometric skeleton computation enabling concurrent product engineering and assembly sequence planning. Computer-Aided Design 43, 1654-1673 (2011) 
10. Demoly, F., Yan, X.-T., Eynard, B., Kiritsis, D., Gomes, S.: Integrated product relationships management: a model to enable concurrent product design and assembly sequence planning. Journal of Engineering Design 23, 544-561 (2012)

11. Gane, V., Haymaker, J.: Design Scenarios: Enabling transparent parametric design spaces 6, 618-640 (2012)

12. Kim, K.J., Sacks, E., Joskowicz, L.: Kinematic analysis of spatial fixed-axis higher pairs using configuration space 6, 279-291 (2001)

13. Li, C.G., Li, C.L., Liu, Y., Huang, Y.: A new C-space method to automate the layout design of injection mould cooling system 6, 811-823 (2012)

14. Miyakawa, S., Shigemura, T.: The Hitachi assemblability evaluation method (AREM). In: Proc. Jpn. Soc. Mech. Eng., Conf. Manuf. Syst. Environlooking Toward 21st Century, pp. 277-282 (1990)

15. Pahl, G., Beitz, W.: Engineering design, a systematic approach, 2nd edn., p. 544. Springer, London (1996)

16. Redford, A., Chal, J.: Design for assembly principles and practice. McGraw-Hill Inc., England (1994)

17. Stone, R., McAdams, D., Kayyalethekkel, V.J.: A product architecture-based conceptual DFA technique. Design Studies 25, 301-325 (2004)

18. Theodosiou, G., Sapidis, N.S.: Information of layout constraints for product lifecycle management: a solid-modelling approach 6, 549-564 (2003)

19. Yamagiwa, Y.: An assembly ease evaluation method for product engineers: DAC. Tech. Jpn. 21 (1988) 